\title{
Dynamical Characteristics Common to Neuronal Competition Models
}

\author{
Asya Shpiro, ${ }^{1}$ Rodica Curtu, ${ }^{3}$ John Rinzel,,${ }^{1,2}$ and Nava Rubin ${ }^{1}$ \\ ${ }^{1}$ Center for Neural Science and ${ }^{2}$ Courant Institute of Mathematical Sciences, New York University, New York, New York; \\ and ${ }^{3}$ Department of Math Analysis and Probabilities, Transilvania University of Brasov, Brasov, Romania
}

Submitted 9 June 2006; accepted in final form 20 October 2006

\begin{abstract}
Shpiro A, Curtu R, Rinzel J, Rubin N. Dynamical characteristics common to neuronal competition models. J Neurophysiol 97: 462-473, 2007. First published October 25, 2006; doi:10.1152/jn.00604.2006. Models implementing neuronal competition by reciprocally inhibitory populations are widely used to characterize bistable phenomena such as binocular rivalry. We find common dynamical behavior in several models of this general type, which differ in their architecture in the form of their gain functions, and in how they implement the slow process that underlies alternating dominance. We focus on examining the effect of the input strength on the rate (and existence) of oscillations. In spite of their differences, all considered models possess similar qualitative features, some of which we report here for the first time. Experimentally, dominance durations have been reported to decrease monotonically with increasing stimulus strength (such as Levelt's "Proposition IV"). The models predict this behavior; however, they also predict that at a lower range of input strength dominance durations increase with increasing stimulus strength. The nonmonotonic dependency of duration on stimulus strength is common to both deterministic and stochastic models. We conclude that additional experimental tests of Levelt's Proposition IV are needed to reconcile models and perception.
\end{abstract}

IN TR O D U C T I O N

Binocular rivalry occurs when two different images are presented to the two eyes. With such ambiguous stimuli, only one of the images is perceived at any given moment, with dominance switching between the two images in a haphazard manner. The average dominance durations are typically a few seconds. Several stimulus parameters have been shown to influence the dynamical characteristics of the perceptual alternations. In particular, increasing the contrast of the rivaling images has been shown to increase the frequency of percept switching, which implies a decrease in the mean dominance times, an observation known as "Levelt's Proposition IV" (Levelt 1968).

Reciprocal inhibition architecture is widely used to describe binocular rivalry and bistable perception in general. The dominant side of the system exerts a strong inhibitory influence on the competing side, so that the latter is suppressed. The switching in dominance between the two sides is realized by a slow negative feedback process, such as spike-frequency adaptation or synaptic depression, that weakens the inhibition either by decreasing the activity of the dominant side or by decreasing the connectivity between the sides and allows the suppressed population to become active. These general principles have been incorporated in numerous mathematical models of binocular rivalry (Blake 1989; Grossberg 1987; Kalarikal

Address for reprint requests and other correspondence: A. Shpiro, Center for Neural Science, New York University, 4 Washington Place, New York, NY 10003 (E-mail: avs203@nyu.edu). and Marshall 2000; Lago-Fernandez and Deco 2002; Laing and Chow 2002; Lehky 1987; Matsuoka 1984; Stollenwerk and Bode 2003; Wilson 2003). We analyzed the effect of varying stimulation strength over a wide range in two models from this family, those by Wilson (2003) and Laing and Chow (2002). These population firing rate models involve two neuronal populations corresponding to the neural representations of the competing percepts. In the model introduced by Laing and Chow (2002) cross-inhibition is realized directly between the two populations. In the model presented by Wilson (2003), separate inhibitory subpopulations are introduced, so that the cross-inhibition acts through a relay stage.

In addition to studying these two models as formulated, we also consider two variations of the model of Laing and Chow (2002). Across these four different models, we implement two types of slow negative feedback processes: one is synaptic depression, which acts by decreasing the effective synaptic connectivity, directly reducing the amount of negative feedback (if in the inhibitory connection), or the amount of positive feedback (if in the excitatory connection) in the system. The second type of process is spike-frequency adaptation, which acts by reducing the excitability of the active neurons in the network, opposing the positive feedback and external input.

We identify the parameter regimes where each model shows behavior that is consistent with Levelt's Proposition IV. In addition, we demonstrate that all the models predict previously unreported types of behavior. Using stimulation strength as the control variable, we focus on its effect on the existence and rate of oscillations. In spite of the differences in architecture and mathematical formulation of the explored models, we find substantial generalities in their behavior. In all models, for very high stimulus strengths the two populations are simultaneously active at a high level. Just below this regime is a range for stimulus strength where the behavior of the system is oscillatory, with the dominance period of each percept decreasing as stimulus strength increases, in accordance with Levelt's Proposition IV (decreasing duration, or DD behavior). However, for input strengths below this range new regimes of behavior are discovered: first, a winner-take-all (nonrivaling steady dominance) behavior appears. Next, as stimulus strength is further reduced, another range of rivalry (oscillatory) behavior appears, but this time with the dominance periods increasing with increasing input (increasing duration, or ID behavior). Finally, at very low input strengths there is again a range where the two populations are simultaneously active, this time at a low level

\footnotetext{
The costs of publication of this article were defrayed in part by the payment of page charges. The article must therefore be hereby marked "advertisement" in accordance with 18 U.S.C. Section 1734 solely to indicate this fact.
} 
that approaches zero with stimulus strength, in agreement with experimental reports of "fusion" (Liu et al. 1992).

Thus rather than showing a monotonic decrease of oscillation period in the entire range of increasing stimulus strength (as Levelt's Proposition IV implies), we find that the period versus stimulus strength dependency is nonmonotonic, passing through an ID regime before it reaches the DD regime (in addition to passing through three other, nonoscillatory regimes). Although the strength of the mutual inhibition can affect the shape of the curve of period versus stimulus strength in quantitative details, the qualitative prediction of this curve being nonmonotonic is very robust. Moreover, the increasing duration behavior exists not only for deterministic models, but also in the presence of noise.

\section{METHODS}

We consider four distinct models of neuronal competition: one (W) by Wilson (2003), the second (LC) by Laing and Chow (2002), and the others (adaptation-LC and depression-LC) are our variations of the LC model. All models are idealized population firing rate models. The network architectures are shown schematically in Fig. 1, $A$ and $B$, respectively. Populations of neurons, corresponding to the left and right boxes in each panel, are characterized by the spatially and short-time-averaged firing rates. The neuronal populations respond to the two competing stimuli presented to the two eyes. The rivalry between dissimilar stimuli representations is realized by reciprocal inhibition (lines with filled circles in Fig. 1), whereas slow processes, such as synaptic depression or self-adaptation provide for the network's oscillatory behavior.

We note the following differences between the models. First, separate inhibitory populations (bottom level boxes in Fig. 1A), are introduced in the W model, so that cross-inhibition between competing populations is indirect contrary to the direct cross-inhibition in the LC, adaptation-LC, and depression-LC models. Second, recurrent excitation (dashed lines with arrows in Fig. $1 B$ ) is included in the LC model, but not in the $\mathrm{W}$, adaptation-LC, and depression-LC models. Third, the input-output (gain) functions for the firing rate have different forms in these models. Finally, the slow processes are implemented differently in the models. Both spike-frequency adaptation and synaptic depression are included in the LC model. Wilson's model and adaptation-LC models contain adaptation only. A depression-LC model contains only synaptic depression. In addition, the dynamics of the slow process variables varies from model to model. In the text below, we further describe the similarities and the differences between the models.
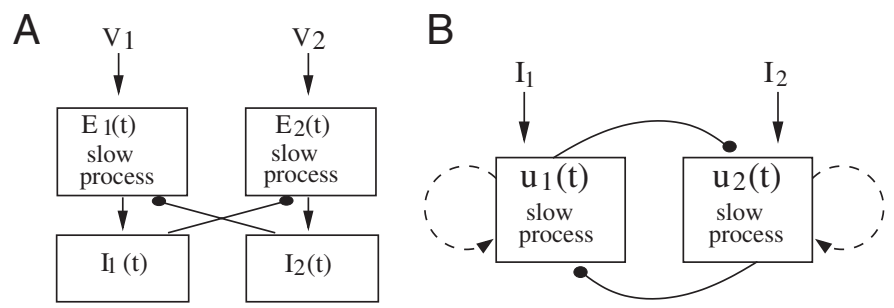

FIG. 1. Network architecture for (A) Wilson's model and $(B)$ Laing and Chow (LC, adaptation-LC, and depression-LC) models. Excitatory and inhibitory connections are represented, respectively, by arrows and filled circles. Dashed lines indicate that the recurrent excitation process is present in the LC model, but is absent in the adaptation-LC and depression-LC models. Original notation of Wilson (2003) and Laing and Chow (2002) is retained: in $A, E_{1,2}$ is the activity of the excitatory populations, $I_{1,2}$ is the activity of the inhibitory populations, and $V_{1,2}$ is the input. In $B, u_{1,2}$ is the population activity and $I_{1,2}$ is the input.
We study the behavior of the network within these models as a function of the stimulus strength, common to both populations, and as a function of the cross-inhibition strength.

Wilson's model (Wilson 2003) is described by the following system of equations

$$
\begin{aligned}
\tau \dot{E}_{1} & =-E_{1}+\frac{100\left(V_{1}-g I_{2}\right)_{+}^{2}}{\left(10+H_{1}\right)^{2}+\left(V_{1}-g I_{2}\right)_{+}^{2}} \\
\tau_{H} \dot{H}_{1} & =-H_{1}+h E_{1} \\
\tau_{1} \dot{I}_{1} & =-I_{1}+E_{1} \\
\tau \dot{E}_{2} & =-E_{2}+\frac{100\left(V_{2}-g I_{1}\right)_{+}^{2}}{\left(10+H_{2}\right)^{2}+\left(V_{2}-g I_{1}\right)_{+}^{2}} \\
\tau_{H} \dot{H}_{2} & =-H_{2}+h E_{2} \\
\tau_{I} \dot{I}_{2} & =-I_{2}+E_{2}
\end{aligned}
$$

Here $V_{i}$ is the input to the population $i(i=1,2), E_{i}(t)$ is the firing rate of the excitatory population $i, H_{i}(t)$ is the adaptation variable, and $I_{i}(t)$ is the inhibitory firing rate. Note that all variables and parameters used are dimensionless. The asymptotic firing rate (the gain function, the second term on the right-hand side of the first and fourth expressions in the Eq. 1 system) is determined by a Naka-Rushton function for positive values of its argument $(V-g I)_{+}$, where $(V-g I)_{+}=V-$ $g I$ if $V \geq g I$ and $(V-g I)_{+}=0$ if $V<g I$ (Naka and Rushton 1966). The following values of the parameters are used: $\tau=20, \tau_{H}=900$, $\tau_{I}=11$, and $h=0.47$, as in the original paper (Wilson 2003). The values of the strength of the cross-inhibition parameter $g$ considered are $0.34,0.42$, and 0.44 . [We assumed there was a typo in Wilson (2003), where $g$ is stated to be 45.0, which is two orders of magnitude larger than it should be to obtain the behavior described there.]

The second model we consider is the Laing and Chow (LC) model (Laing and Chow 2002). The mean-field dimensionless equations for the LC model are the following

$$
\begin{aligned}
\dot{u}_{1} & =-u_{1}+f\left(\alpha u_{1} g_{1}-\beta u_{2} g_{2}-a_{1}+I_{1}\right) \\
\tau_{a} \dot{a}_{1} & =-a_{1}+\phi_{a} f\left(\alpha u_{1} g_{1}-\beta u_{2} g_{2}-a_{1}+I_{1}\right) \\
\tau_{d} \dot{g}_{1} & =1-g_{1}-g_{1} \phi_{d} f\left(\alpha u_{1} g_{1}-\beta u_{2} g_{2}-a_{1}+I_{1}\right) \\
\dot{u}_{2} & =-u_{2}+f\left(\alpha u_{2} g_{2}-\beta u_{1} g_{1}-a_{2}+I_{2}\right) \\
\tau_{a} \dot{a}_{2} & =-a_{2}+\phi_{a} f\left(\alpha u_{2} g_{2}-\beta u_{1} g_{1}-a_{2}+I_{2}\right) \\
\tau_{d} \dot{g}_{2} & =1-g_{2}-g_{2} \phi_{d} f\left(\alpha u_{2} g_{2}-\beta u_{1} g_{1}-a_{2}+I_{2}\right)
\end{aligned}
$$

Here $u_{i}$ represents the averaged activity of population $i$, normalized so that its maximum value is $1 ; a_{i}$ is the population adaptation variable, with timescale $\tau_{a}$; and $g_{i}$ is the synaptic depression variable, with timescale $\tau_{d}$. The gain function $f$ is taken to be the Heaviside step function, i.e., $f(x)=1$ for $x \geq 0$ and $f(x)=0$ for $x<0$. The positive inputs to the gain functions consist of the external stimulus and the recurrent excitation term proportional to $u_{i}$ and scaled by $\alpha$. The negative inputs are the cross-inhibition term of strength $\beta$ and the adaptation term. Both recurrent excitation and cross-inhibition terms are multiplicatively reduced by the synaptic depression variables. Note that here, adaptation is incorporated in a manner different from that in the Wilson model: it shifts the threshold of the gain function, but does not change its slope. We will distinguish between the two types of slow negative feedback: subtractive, when the threshold of the gain function is shifted, and divisive, when the slope changes. The former is realized in the LC and adaptation-LC models' adaptation process and the latter in both the LC and depression-LC models' synaptic depression process and W model's adaptation. We further discuss the consequences of this distinction in later sections. The following values of the parameters are used: $\alpha=0.35, \phi_{a}=\phi_{d}=$ $0.6, \tau_{a}=20$, and $\tau_{d}=40$, as in the original paper (Laing and Chow 
2002). The strength of the cross-inhibition $\beta$ considered is $0.01,0.2$ (as in the original paper), and 0.7 .

To investigate the role of the slow processes in the system's behavior, we modify the LC model to include only the synaptic depression or only the spike-frequency adaptation. To compare the dynamics of the LC system with and without recurrent excitation, we omit the recurrent excitation term in the gain function inputs. In addition, linear (unsaturating) gain for slow process variables is considered, unlike in the original LC model.

The model with synaptic depression only (depression-LC) is described by the following system of equations

$$
\begin{aligned}
\dot{u}_{1} & =-u_{1}+f\left(-\beta u_{2} g_{2}+I_{1}\right) \\
\tau_{d} \dot{g}_{1} & =1-g_{1}-\gamma u_{1} g_{1} \\
\dot{u}_{2} & =-u_{2}+f\left(-\beta u_{1} g_{1}+I_{2}\right) \\
\tau_{d} \dot{g}_{2} & =1-g_{2}-\gamma u_{2} g_{2}
\end{aligned}
$$

Instead of the step function, we consider a sigmoid gain, $f(x)=$ $1 /\{1+\exp [-(x-\theta) / k]\}$, where $1 / k$ defines its slope and $\theta$ defines the threshold. Because the depression variable in this model works to decrease the slope of the gain function, the depression-LC model is, in fact, similar to the $\mathrm{W}$ model, in spite of the apparently different mathematical implementation. We classify this model as having a divisive slow process. The following values of parameters are used: $k=0.1, \theta=0.1, \gamma=0.3$, and $\tau_{d}=150$. The values of the parameter $\beta$ are $0.47,0.53$, and 0.6 .

Finally, the model with spike-frequency adaptation only (adaptation-LC) is expressed in the following system of equations

$$
\begin{aligned}
\dot{u}_{1} & =-u_{1}+f\left(-\beta u_{2}-g a_{1}+I_{1}\right) \\
\tau_{a} \dot{a}_{1} & =-a_{1}+u_{1} \\
u_{2} & =-u_{2}+f\left(-\beta u_{1}-g a_{2}+I_{2}\right) \\
\tau_{a} \dot{a}_{2} & =-a_{2}+u_{2}
\end{aligned}
$$

where $g$ is the strength of the adaptation. The gain function is again a sigmoid. This model belongs to a class of models with a subtractive slow process. Parameters used are: $k=0.1, \theta=0.2, g=0.5, \tau_{a}=$ 100 , and the values of $\beta$ are $0.41,0.9$, and 1.1 .

Table 1 summarizes the key features of the models that we are considering.

Random behavior in all network models is simulated by introducing a stochastic forcing term: in addition to external stimuli $I_{i}$, both populations receive independent input noise $n_{i}$. It is defined by the following equation

$$
\dot{n}_{i}=-\frac{n_{i}}{\tau_{n}}+\sigma \sqrt{\frac{2}{\tau_{\mathrm{n}}}} \eta(t)
$$

where $\eta(t)$ is white noise with zero mean and unit variance. The resulting $n_{i}$ is an exponentially filtered white noise with standard deviation $\sigma$ and timescale $\tau_{n}$ (Risken 1989). This choice of noise models the synaptic filtering of inputs to a neuron (Moreno-Bote and Parga 2004). The timescale for the noise is chosen to be faster than the timescale of the slow negative feedback processes in the system, $\tau_{n}=$ 10. The strength of the noise is taken to be $\sigma=0.03$

We consider population $i$ to be dominant and population $j$ to be suppressed if $u_{i}>u_{j}$. The strength of the input to the system $\left(V_{i}\right.$ in the $\mathrm{W}$ model and $I_{i}$ in the LC, adaptation-LC, and depression-LC models) is assumed to increase monotonically with the stimulus strength (typically, the contrast).

The deterministic models' equations were implemented within Mathematica software, using the predictor-corrector Adams method with precision of $10^{-8}$. Stochastic equations were implemented within $\mathrm{C}$ programming language using forward Euler's method. The GNU scientific library was used to obtain white noise distribution. Bifurcation diagrams were computed using a freely available software package XPPAUT by G. B. Ermentrout (http://www.math.pitt.edu/ $\sim$ bard/bardware).

\section{RES U L T S}

Unless explicitly stated otherwise, all results presented below are for deterministic models.

\section{Spike-frequency adaptation as a divisive slow process}

We start our analysis from the original W model $(E q .1$ system). In Fig. 2, the time courses of the network activity are presented, for different values of the stimulus strength, equal in both neuronal populations. Figure $2, A-E$ shows $E_{1}(t)$ and $E_{2}(t)$, solid and dashed lines, for five values of input: $V_{1}=$ $V_{2}=38,15,6,3.5$, and 2 . Values of the other parameters are taken from the work of Wilson (2003) (see METHODS). The values of $V_{1}$ and $V_{2}$ considered here correspond to five qualitatively different regimes of the network's dynamics. In regime I (Fig. 2A) the two populations are simultaneously active at a high level: $E_{1}(t)$ equals $E_{2}(t)$ as $t$ becomes larger than the longest timescale in the system, $\tau_{H}$, so that the transients have decayed. Regime II (Fig. 2B) is oscillations, when the two populations take turns in being active. In regime III (Fig. 2C) we find winner-take-all behavior, when, depending on the initial conditions, only one population remains active indefinitely, whereas the other remains silent. Regime IV (Fig. 2D) is again oscillatory. As demonstrated below, regimes II and IV

\begin{tabular}{|c|c|c|c|c|}
\hline \multirow[b]{2}{*}{ Model } & \multicolumn{4}{|c|}{ Feature } \\
\hline & Architecture & Adaptation & Depression & Gain Function \\
\hline Wilson (W) & $\begin{array}{l}\text { Direct and indirect } \\
\text { cross-inhibition, no self-excitation }\end{array}$ & Yes (divisive) & No & Naka-Ruston \\
\hline Laing and Chow (LC) & Direct cross-inhibition, self-excitation & Yes (subtractive) & Yes (divisive) & Heaviside \\
\hline Adaptation-LC & Direct cross-inhibition, no self-excitation & Yes (subtractive) & No & Sigmoid \\
\hline Depression-LC & Direct cross-inhibition, no self-excitation & No & Yes (divisive) & Sigmoid \\
\hline
\end{tabular}
differ in how the oscillations' period depends on the stimulus strength. Finally, regime V (Fig. 2E) corresponds to what has been termed "fusion" in experimental works: simultaneous, low level of activity of the two populations (Liu et al. 1992).

As seen in Fig. 2, the behavior of the $\mathrm{W}$ model depends on the value of the external input to the system. We varied the input strength, common to both neuronal populations, between

TABLE 1. Key features of the considered models

Varied parameters are stimulus strength $\left(V_{i}\right.$ in the W model and $I_{i}$ in the LC, adaptation-LC, and depression-LC models) and strength of cross-inhibition ( $g$ in the $\mathrm{W}$ model and $\beta$ in the LC, adaptation-LC, and depression-LC models). 
0 and 40, to classify the system's dynamics according to whether oscillatory (rivaling) behavior exists and how the oscillations' period depends on the input. Figure $3 A$ summarizes results of the simulations. We observe the behavior of the system and plot the period of oscillations of network as a function of input strength (if oscillations are present).

There are five distinct regions in the period versus stimulus strength diagram. For input values $>34$ (regime I), the system does not oscillate. Instead, both populations are active at an identically high level. For input strengths between 7.4 and 34 (regime II), rivaling behavior exists and the period of oscillations decreases as the stimulus strength increases. We call regime II a decreasing duration (DD) regime. Further decrease in the stimulus strength (between 4.2 and 7.4, regime III) leads to disappearance of the oscillatory behavior. From the observations of the time course of the system (Fig. 2C) we conclude that regime III is a winner-take all regime, when one population is active and the other is inactive indefinitely (note that the model does not contain noise). Whether population 1 or 2 is
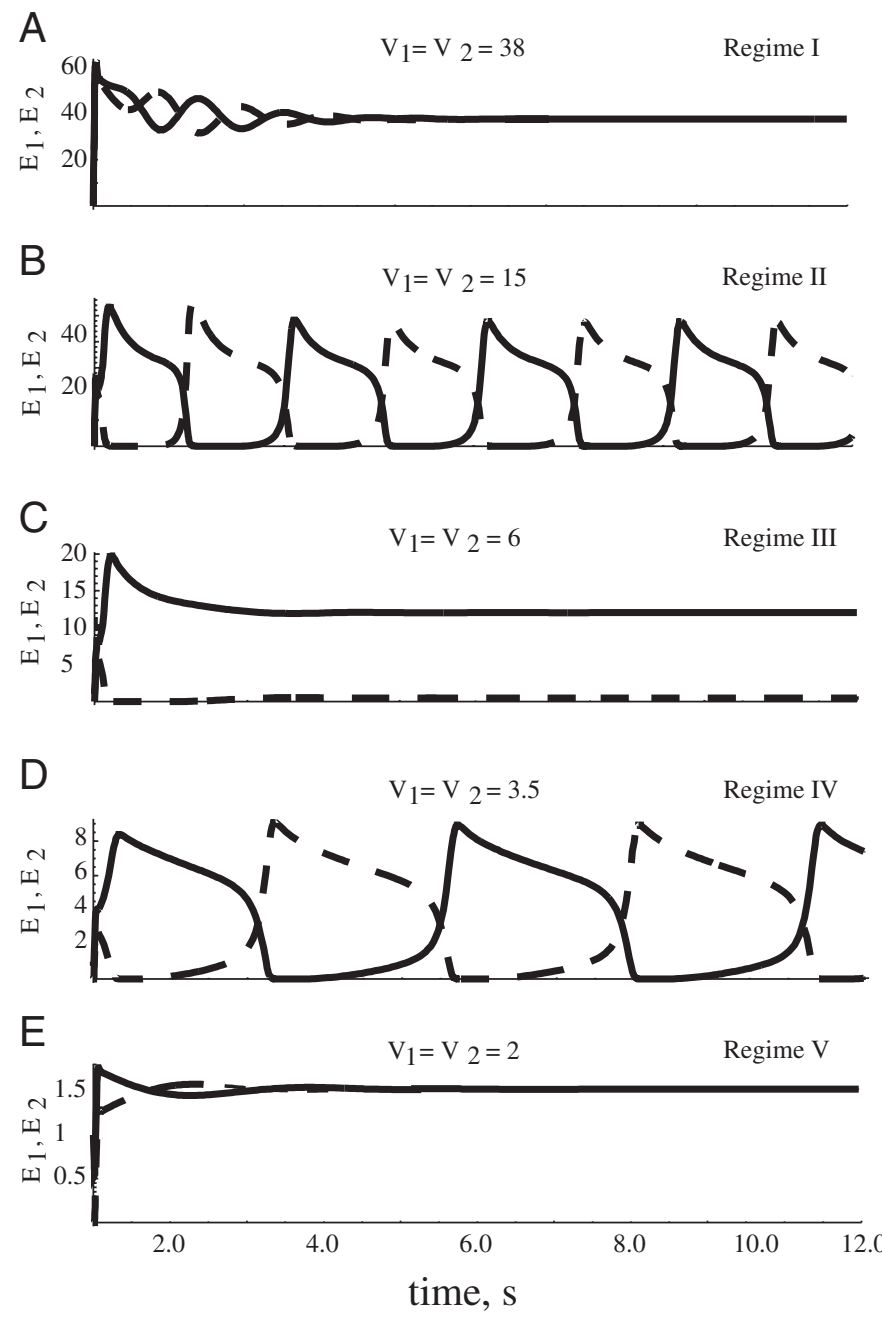

FIG. 2. Time course of activity and for the 2 neuronal populations (solid and dashed lines correspondingly) in Wilson's model in 5 different dynamical regimes: simultaneous activity at high level at $V_{1}=V_{2}=38(A)$, oscillations at $V_{1}=V_{2}=15(\mathrm{~B})$, winner-take-all at $V_{1}=V_{2}=6(\mathrm{C})$, oscillations at $V_{1}=$ $V_{2}=3.5(D)$, and simultaneous activity at low levels (fusion) at $V_{1}=V_{2}=2$ $(E)$. Value of the inhibition strength parameter $g$ used is 0.44 . For values of the other parameters, see METHODS.

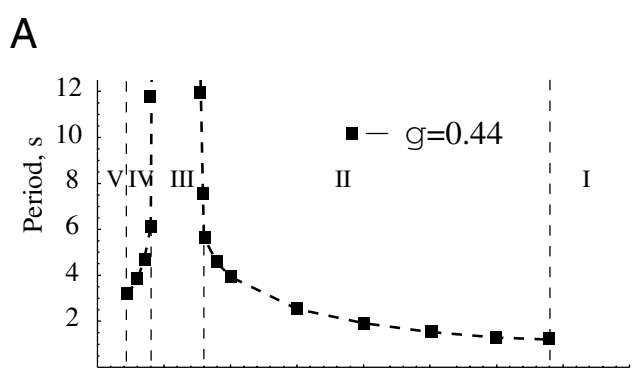

B

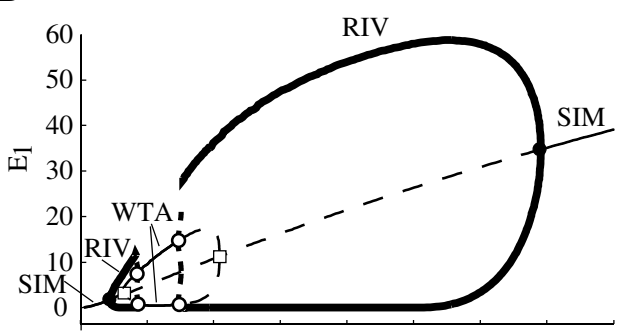

C

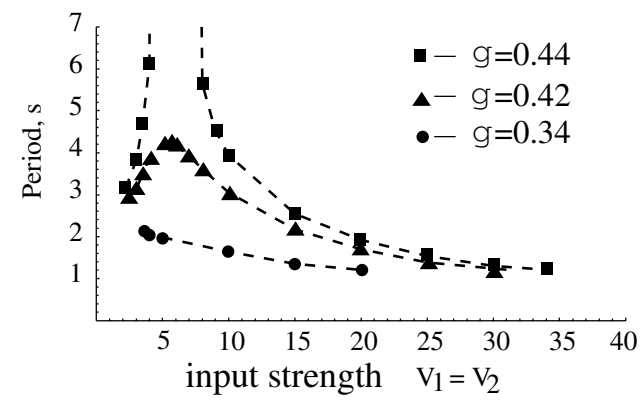

FIG. 3. Five regions of dynamical behavior for Wilson's model. A: period $T$ of the network's rivalry oscillations as a function of input strength $(A)$ for the original Wilson model, which implements adaptation in a divisive fashion. Regime II corresponds to decreasing duration behavior, and regime IV to increasing duration behavior. $B$ : bifurcation diagram shows response amplitude for the model's solutions. Branched heavy solid curves (labeled RIV) correspond to the maximum and minimum activity during rivalry oscillations; the simultaneous activity solution corresponds to the (thin) monotonic curve labeled SIM; the winner-take-all state is double-branched (thin curve, labeled WTA) and shows the activity of the dominant units (top branch) and suppressed units (bottom branch). A solution state is unstable (and physically unrealizable) where a curve is dashed; bifurcation points, where solutions emerge or disappear, are indicated by circles or squares. $C$ : rivalry period is plotted vs. stimulus strength for different values of inhibition strength parameter: $g=0.44$ (square symbols), 0.42 (triangles), and 0.34 (circles); $g=0.44$ in $A$ and $B$. For values of the other parameters, see METHODS.

active is determined by the initial conditions. Oscillations resume as the stimulus strength becomes even smaller (between 2.2 and 4.2, regime IV). Unlike in regime II, however, here the period of oscillations increases as the stimulus strength increases. We call regime IV an increasing duration (ID) regime. Finally, for inputs $<2.2$ (regime V), rivaling behavior again ceases to exist and fusion at a low level of activity is realized. The presence of the winner-take-all regime was observed in the original report by Wilson, albeit in a different context. The presence of the ID regime is a new prediction that emerges from our analysis of the $\mathrm{W}$ model.

Properties of the system's states in the different regimes can be summarized with a compact graphical description (bifurcation diagram) by plotting response amplitude versus stimulus 
strength (Fig. 3B). The diagram allows us to understand how the system's behavior changes from one regime to the next as the stimulus $\left(V=V_{1}=V_{2}\right)$ is varied, complementing the information in Fig. $3 A$ that shows only the rivaling behavior. First, we focus on the simultaneous activity state for strong stimulus (regime I) indicated by SIM in Fig. 3B. Its amplitude (firing rate) decreases with decreasing $V$ as one expects. According to the curve this state exists (as a solution to the system of differential equations in Eq. 1) for the full range of $V$. However, we know that it gives way to oscillations as $V$ decreases below a critical value, when entering regime II. This occurs because, even though the simultaneous activity solution exists, it becomes unstable (as a solution to the differential equations) for intermediate values of $V$ (where the curve is dashed). It corresponds, in a practical sense, to a nonsustainable state, i.e., any small random perturbations will be amplified and the system will move to the stable oscillatory or WTA solution. As $V$ passes through a critical value (filled circle) the simultaneous activity solution changes character from a stable fixed point with damped oscillatory behavior to a growing oscillation. At this point, the rivaling state emerges, appearing first with small oscillation amplitude (a Hopf bifurcation; Guckenheimer and Holmes 2002). The upper and lower tines of the forked solid curve (labeled RIV) represent the peak and trough of the activity during a cycle. The winner-take-all solution emerges from the (unstable) simultaneous activity solution by a different type of bifurcation. Here, the control state (SIM) loses or gains a mode of instability and the emergent steady state actually involves two possible states (open square). (By symmetry, either population 1 or 2 can be dominant with the other suppressed.) Here the WTA state is itself not stable at emergence (shown dashed). However, following along the WTA branch we see that stabilization occurs (open circle). The scenario more or less repeats in the opposite sequence as we continue to decrease $V$; the solution states of WTA and rivalry disappear as the low-activity SIM state, or fusion, becomes the only stable state for small stimulus strength.

\section{Change of the cross-inhibition strength leads to qualitative changes in model dynamics}

To investigate how the model's behavior depends on the choice of parameters, we first consider the effect of the time constants of the slow negative feedback processes. In the $\mathrm{W}$ model, as in all models considered below, increase in $\tau_{H}\left(\tau_{a}\right.$ or $\tau_{d}$ in the LC, adaptation-LC, and depression-LC models), leads to a proportional increase in the oscillations' period. Transitions between regimes of different behavior occur at bifurcation points in the bifurcation diagrams. These points do not change as the time constants of the slow processes change, as long as these time constants are sufficiently large. The existence of oscillations and the qualitative behavior of the oscillations' period as a function of the stimulus strength are not influenced by the time constants of the slow processes.

We varied the strength of the cross-inhibition parameter $g$ to see whether it influences the dynamics of the $\mathrm{W}$ model. In Fig. $3 C$ we plot the oscillation period $T$ as a function of input strength for three values of $g: g=0.44$ (square symbols, same as in Fig. $3 A$, although notice the change in the scales of the graphs in $A$ and $C$ ), $g=0.42$ (triangle symbols), and $g=0.34$ (circles). We observe that as the inhibition strength decreases from 0.44 to 0.42 , the winner-take-all regime (regime III) disappears. However, the period versus stimulus strength dependency remains nonmonotonic, with an increasing duration regime present. Further decrease in the inhibition strength (to 0.34 ) leads to the disappearance of the ID regime, so that only the DD oscillatory regime remains, bounded by two simultaneous activity regimes, with a low-activity level for weak stimuli and a high-activity level for strong stimuli. Note, however, that as $\mathrm{g}$ decreases, alongside the disappearance of the ID regime there is a dramatic decrease in the effect of stimulus strength on durations in the DD regime, resulting in a limited range of observable oscillation periods. Furthermore, whereas for small values of $g$ the period versus stimulus strength dependency is monotonic, a $25-30 \%$ increase in the inhibition strength results in a qualitative change in the dynamics and a previously unreported type of behavior: nonmonotonic, and even discontinuous, period versus stimulus strength dependencies. We will proceed to show that similar behavior is observed in the other models of neuronal competition.

\section{Synaptic depression as a divisive slow process}

As mentioned in METHODS, the system that most closely resembles the $\mathrm{W}$ model among the versions of the Laing and Chow model we studied is the depression-LC model (Eq. 3 system). In both models, the slow process variables affect the slope of the gain function for the activity variable. The apparent difference between these models is the absence of separate inhibitory populations in the depression-LC model; they are present in the $\mathrm{W}$ model.

For depression-LC model we vary the strength of the input, identical to both populations, $I_{1}=I_{2}$, and plot the period of the oscillations of the network activity in the input ranges where they exist, for three values of the cross-inhibition strength $\beta$ (Fig. $4, A, C$, and $E$ ). For the larger value of $\beta$ ( $\beta=0.6$, Fig. $4 A$ ), we observe the same types of behaviors as in the $\mathrm{W}$ model: simultaneous activity at high input, oscillations with the period decreasing as input strength increases, winner-take-all regime as input strength decreases further, followed by the increasing duration oscillatory regime and fusion at the lowactivity level. In Fig. $4 E$, the results of the simulations for $\beta=$ 0.47 are presented. For this value of the cross-inhibition, the rivaling regime exists, with the period of oscillations decreasing with increasing stimulus strength, bounded by two simultaneous activity regimes, at high- and low-activity levels. Note that here, too, the range of observed periods is small compared with that found for larger $\beta$ values. At an intermediate value $\beta=0.53$ (Fig. $4 C$ ), whereas the winner-take-all regime disappears, the dependency of period of oscillations on input strength is still nonmonotonic.

The bifurcation diagrams of Fig. 4, $B, D$, and $F$ represent the underlying skeletons for the solution states for these three values of $\beta$. Figure $4 B$ is qualitatively like the corresponding description (Fig. $3 B$ ) for the $\mathrm{W}$ model when inhibition is not reduced. In Fig. 4, $D$ and $F$ we find the simpler scenario in which simultaneous activity state or rivalry are the only attracting states; rivalry emerges and disappears by Hopf bifurcations.

The intermediate case (Fig. 4D) suggests how the WTA behavior disappears. Along the corresponding WTA solution 
A

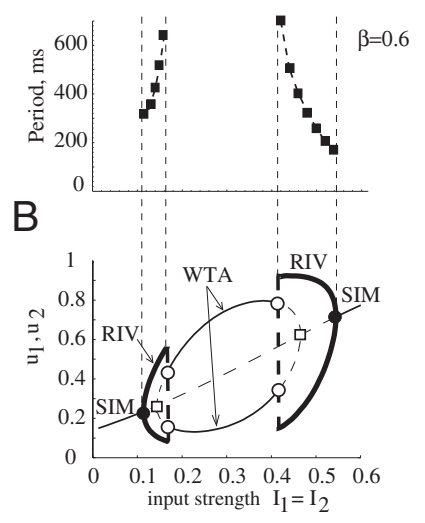

C

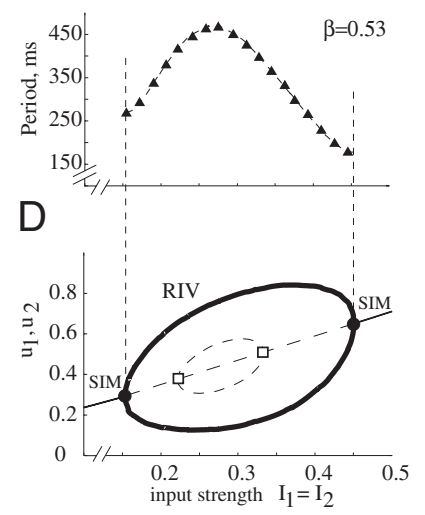

E

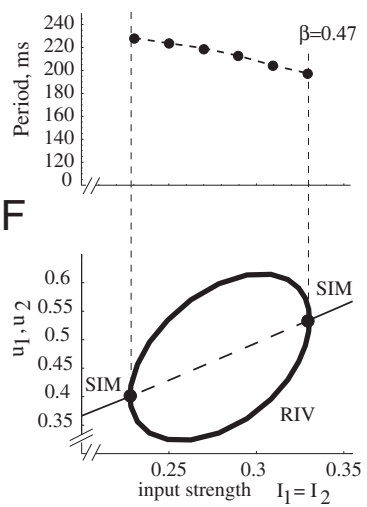

FIG. 4. Period $T$ of network oscillation vs. input strength and bifurcation diagrams for the depression-LC. Inhibition strength parameter is $\beta=0.6(A$ and $B), \beta=0.53(C$ and $D)$, and $\beta=0.47(E$ and $F)$. For values of the other parameters, see METHODS. Line types and symbols in the bifurcation diagrams are in the same format as in Fig. $3 B$. branch the dominant and suppressed activity levels approach each other as $\beta$ decreases from 0.6 , and at the same time the range of input strengths where the stable WTA exists shrinks. Beyond some critical value of $\beta$ the WTA branch has shrunk enough so that stable WTA disappears. The decreasing duration and increasing duration rivalry branches are no longer split but they now form a continuous branch. However, the rivalry alternations are still influenced by the "ghost" of the WTA steady states, as represented by the dashed branch in Fig. 4D. This remnant of the WTA branch is now unstable. There is an $I$-range where the ghost is effective to induce long-period rivalry; presumably the rivalry trajectory visits a region of phase space near to the ghost and slowly moves there. This leads to the nonmonotonic dependency of period on stimulus intensity of Fig. $4 C$. This profile is the continuous extension of the case from Fig. $4 A$ where the period from left and right became unbounded. Eventually (for even smaller $\beta$ ) the ghost is gone and the possibility for the period to vary monotonically with $I$ is realized.

\section{Spike-frequency adaptation as a subtractive slow process}

Next, we performed simulations in the original Laing and Chow (2002) model with recurrent excitation, spike-frequency adaptation, and synaptic depression (LC model, Eq. 2 system) with the parameters identical to those in their paper (Laing and Chow 2002; see METHODS), but varying the cross-inhibition strength parameter $\beta$ (Fig. $5 A$ ). We find that for their value of $\beta=0.2$, the dependency of the period of oscillations $T$ on the stimulus strength is, in fact, nonmonotonic, with an increasing duration regime at input strengths $<0.25$ (line with triangles in Fig. 5A); this regime was not reported in their paper. Note that in the Laing and Chow paper the values of the stimulus strength are rescaled to compare the simulations with the predictions of their spiking model. As in the models discussed earlier, increase in the inhibition strength leads to the appearance of the winner-take-all regime, where rivalry does not exist (illustrated in Fig. $5 A$ for $\beta=0.7$, lines with squares). Decreasing inhibition strength (to a very small value of 0.01 in the case of the parameters in the LC model) leads to what appears to be only a DD branch for period versus stimulus strength (line with circles in Fig. 5A). However, this dependency is still nonmonotonic on a finer scale.

Finally, we considered the adaptation-LC model (Eq. 4 system). In this system, as in the all systems discussed earlier, change in the cross-inhibition strength leads to the qualitative changes in the oscillation period versus stimulus strength dependency (Fig. 5B). For large values of the inhibition strength, this dependency is discontinuous, with the winnertake-all regime for the intermediate values of the input strength and the increasing duration regime for smaller values of input (as shown by the lines with squares for $\beta=1.1$ in Fig. $5 B$ ). As $\beta$ decreases, the period versus input dependency becomes continuous, but remains nonmonotonic (as illustrated by the line with triangles for $\beta=0.9$ in Fig. $5 B$ ). Further decrease in the value of the inhibition strength again leads to what looks like the decreasing-duration-only behavior, but, in fact, it is not (Fig. $5 B$, line with circles for $\beta=0.41$ ). It can be shown analytically in the particular case of the system described by Eq. 4 that if the DD regime of the period versus input dependency is present, then the ID regime must also be present (R. Curtu et al., unpublished observations).
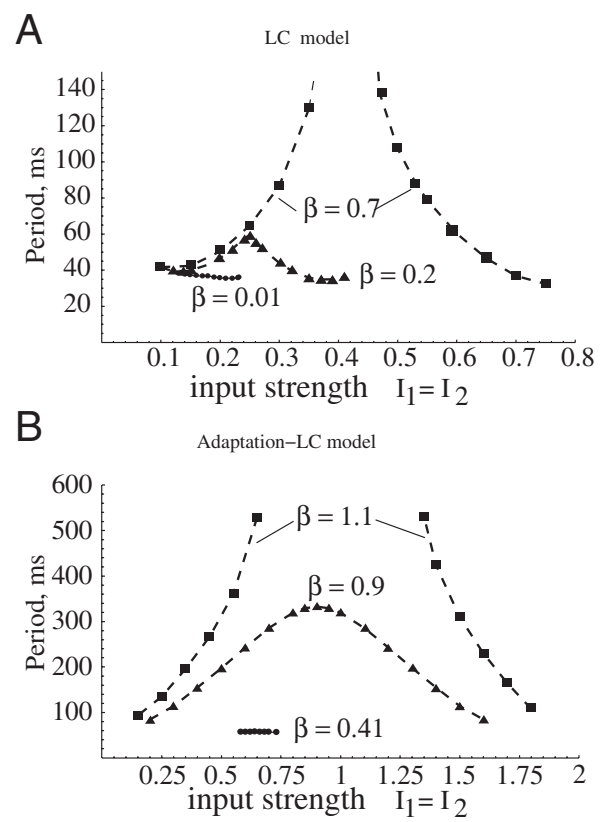

FIG. 5. Period of oscillations $T$ as function of input strength for $(A)$ the LC model and $(B)$ the adaptation-LC model. In both models, adaptation is implemented in a subtractive fashion. Values of the cross-inhibition strength parameter $\beta$ in $A$ are 0.7 (square symbols), 0.2 (triangles), and 0.01 (circles). In $B$, the values of $\beta$ are 1.1 (squares), 0.9 (triangles), and 0.41 (circles). For values of the other parameters, see METHODS. 
Release and escape as mechanisms for generating oscillations in neuronal competition models

During alternating oscillations, the two populations take turns being active. When dominant, a population experiences accumulating slow negative feedback, whereas feedback recovers when a population is suppressed. By intuitive reasoning, a switch in dominance may occur in either of two ways, depending on whether accumulation of or recovery from feedback is more influential in tipping the balance of net input to a population. We say that "release" is the mechanism when the switch-determining factor is accumulating negative feedback that overcomes the stimulus to an active population. In this case the active population loses control, its activity drops, and it no longer suppresses the other population. Alternatively, switching takes place by "escape" when in the inhibited population feedback recovers sufficiently so that the net input changes from negative (suppressing) to positive (activating). Now, the "down" population regains control, bounding into activity and causing the other population to become inhibited. In release, one might expect to observe more readily a decline in activity of the dominant population compared with a rise in the suppressed population, and the opposite behavior in escape. However, behavior of the populations' activities before the switch will depend on the shapes of the input-output functions. For example, in the case of Heaviside gain functions, discussed in the following text, transitions are abrupt in both dominant and suppressed populations.

If we suppose that slow negative feedback operates over a finite range (say, because of saturation) we can understand that release occurs for lower-stimulus ranges rather than for higherstimulus ranges (where adaptation might not be able to overcome the stimulus) and conversely for escape. These mechanisms of release and escape thus underlie the ID and DD behaviors, respectively. We describe these correspondences for a simplified model in the next paragraphs. The terms "release" and "escape" were previously used to describe inhibitionmediated rhythmic patterns in other neuronal systems (see Wang and Rinzel 1992 and references therein).

We will use the adaptation-LC model (described by the Eq. 4 system) to explain the mechanistic basis for the decreasing dominance (DD) and increasing dominance (ID) regimes in the period versus stimulus strength dependency. Here, for simplicity, we take the gain function to be a Heaviside step function, i.e., $f(x)=1$ for $x>0$ and $f(x)=0$ for $x<0$. As a consequence, the fast activity variables $u_{i}$ can only have the value of one (active population) or zero (inactive population). The slow adaptation variable $a_{i}$ of an active population grows with timescale $\tau_{a}$, whereas the adaptation variable of an inactive population decreases with the same timescale.

As follows from $E q .4$, the switch between $u_{i}=1$ and $u_{i}=$ 0 occurs when the value of the gain function changes from 1 to 0 , i.e., when the net input to a population, the value of the gain function argument, $\left(-\beta u j-g a_{i}+I_{i}\right)$, changes sign from positive to negative. In the release mechanism, the adaptation variable of the "up" population increases so much that the sign of the gain function argument for this population changes from positive to negative. This population becomes inactive, therefore releasing the "down" population from inhibition; the latter's net input then rapidly becomes positive, bringing this population "up." The greater the input $I_{i}$, common to both populations, the longer it takes for the negative-feedback adaptation variable of the "up" population to grow enough to change the net input (the argument of the gain function) from positive to negative and to produce switching, and the longer this population remains active. In the adaptation-LC model, a stronger stimulus means longer dominance time, or ID behavior during the oscillations realized by release mechanism. Further, we can see that release can occur only if $I_{i}$ is not large. Because $u_{j}=0$ and $a_{i}$ cannot exceed 1 , the sign change and therefore release is precluded for $I_{i}>g$. Thus ID behavior is realized in the low-stimulus regime.

In contrast, in the escape mechanism, the adaptation variable of the "down" population decreases so much that the net input to this population changes from negative to positive. This population becomes active, or escapes from inhibition, and promptly pushes the other population down. The greater the stimulus, the less negative is the net input to the "down" population and the less time it takes for the recovering (decreasing) adaptation to change the sign of the gain function argument from negative to positive and to produce switching. In the adaptation-LC model, stronger input means shorter dominance time, or DD behavior during the oscillations produced by the escape mechanism. Escape can occur only if $I_{i}$ is large enough. Because $u_{j}=1$ and $a_{i}$ cannot decrease below 0 , $I_{i}$ has to be $>\beta, I_{j}>\beta$, to allow for the sign change. DD behavior is realized in the higher stimulus regime.

For intermediate stimulus strengths, rivalry alternations or winner-take-all behavior may occur, depending on parameter values (e.g., compare in Fig. $5 B$ the cases $\beta=0.9$ and $\beta=$ 1.1). In the case of alternations the distinction between release and escape may blur unless the gain function is very steep. For the WTA regime (in the Heaviside case) we argue as follows. From Eq. 4, the range of the adaptation variable is restricted; $a_{i}$ can vary only between 0 and 1 . Combinations of parameter values $\left(\beta, g\right.$, and $\left.I_{i}\right)$ exist when the variation of $a_{i}$ through this range is not enough to change the sign of the gain function argument and oscillations are impossible. If, say, $\beta$ is sufficiently large, then either release or escape is precluded for a range of $I$-values that is intermediate between the ID and DD branches. The net input to the active population $i,\left(-\beta^{*} 0-\right.$ $\left.g a_{i}+I_{i}\right)$ varies between $\left(I_{i}-g\right)$ and $I_{i}$, so it is always positive if $I_{i}>g$. Similarly, the net input to the inactive population $j$, $\left(-\beta^{*} 1-g a_{j}+I_{j}\right)$ varies between $\left(-\beta-g+I_{j}\right)$ and $(-\beta+$ $\left.I_{j}\right)$, so it is always negative if $I_{j}<\beta$. In the case of large inhibition strength $(\beta>g)$, the range of the inputs exist $(g<$ $I_{i}<\beta$ ) such that neither the net input to the active population nor that to the inactive population can change sign, and the switch between activities is not possible. This range corresponds to the WTA regime.

Similar arguments can be made in the case of the depression-LC model. If a sigmoid is used as a gain function, both ID and DD branches of the period versus stimulus strength dependency typically appear (see Fig. 3A). Both release and escape mechanisms of producing oscillations are realized. The depression-LC model with the Heaviside gain function is a special case. The ID branch does not appear. Only the DD branch appears and only the escape mechanism is realized, explained by the fact that the negative feedback term is absent in the gain function argument of the active population, thereby precluding release. 
The release, escape, and winner-take-all behaviors that we described earlier are compatible with the regimes seen on the bifurcation diagrams like those that we presented for the sigmoid gain functions (see Figs. $3 B$ and $4 B$ ). A more detailed and general analysis of the nonlinear differential equations describing the models is possible but is beyond the scope of this paper.

\section{Nonmonotonic duration dependency, but not winner-take-all} behavior, is robust in the presence of noise

We induce random behavior in the firing rate network models by introducing a stochastic (zero mean) component to the external input (see METHODS). We illustrate the effects of noise for the case of the depression-LC model (similar results were obtained for all the other models). The model's parameters were taken such that the noise-free version exhibited all five regimes of behavior as described earlier (Fig. 4A). In the presence of noise, dominance durations vary randomly. The major effect is that the winner-take-all regime of behavior ceases to exist because the random input precludes the possibility of one percept dominating forever. Consequently, the mean period (over long runs) versus stimulus strength now forms a continuous curve and, importantly, the nonmonotonic dependency of period persists (Fig. 6A). Thus whereas winnertake-all disappears, the increasing duration behavior for some stimulus range does not: both ID and DD branches are seen in the period versus stimulus strength dependency. With noise, dominance switching occurs more frequently on average (compared with the noise-free periods, shown in solid, from Fig. $4 A$ ). Figure $6 B$ shows a typical time course of the network activity in the presence of noise for the input strength $I_{1}=I_{2}=$ 0.3 . We also computed the distribution of random periods in this model for that value of input strength (Fig. 6C). It appears to be unimodal and skewed, with a longer tail at long periods, consistent with experimental results (Fox and Herrmann 1967; Logothetis et al. 1996).

\section{I S C U S S I ON}

We considered several neuronal competition models to describe oscillatory dynamics during binocular rivalry. We focused on the behavior of these models as we vary the stimulus (input) strength assumed equal for both populations. We used previously published parameters of the original $\mathrm{W}$ and $\mathrm{LC}$ models, and similar parameters in the adaptation-LC and depression-LC models. We varied, as a system parameter, the strength of cross-inhibition in the network. Here is a summary of our main findings.

\section{Five modes of behavior}

In all considered models, we observed five distinct regimes of behavior in different ranges of stimulus strength. Over a range of strong stimulus strengths the period of rivaling oscillations decreases with increasing input and then for very strong stimuli alternation is lost — both populations are steadily, highly, and identically active. For intermediate values of the stimulus strength, a winner-take-all regime is present. Another oscillatory regime exists for smaller inputs, with the period of oscillations increasing as input increases. At very low stimulus strengths the system goes into fusion with both populations
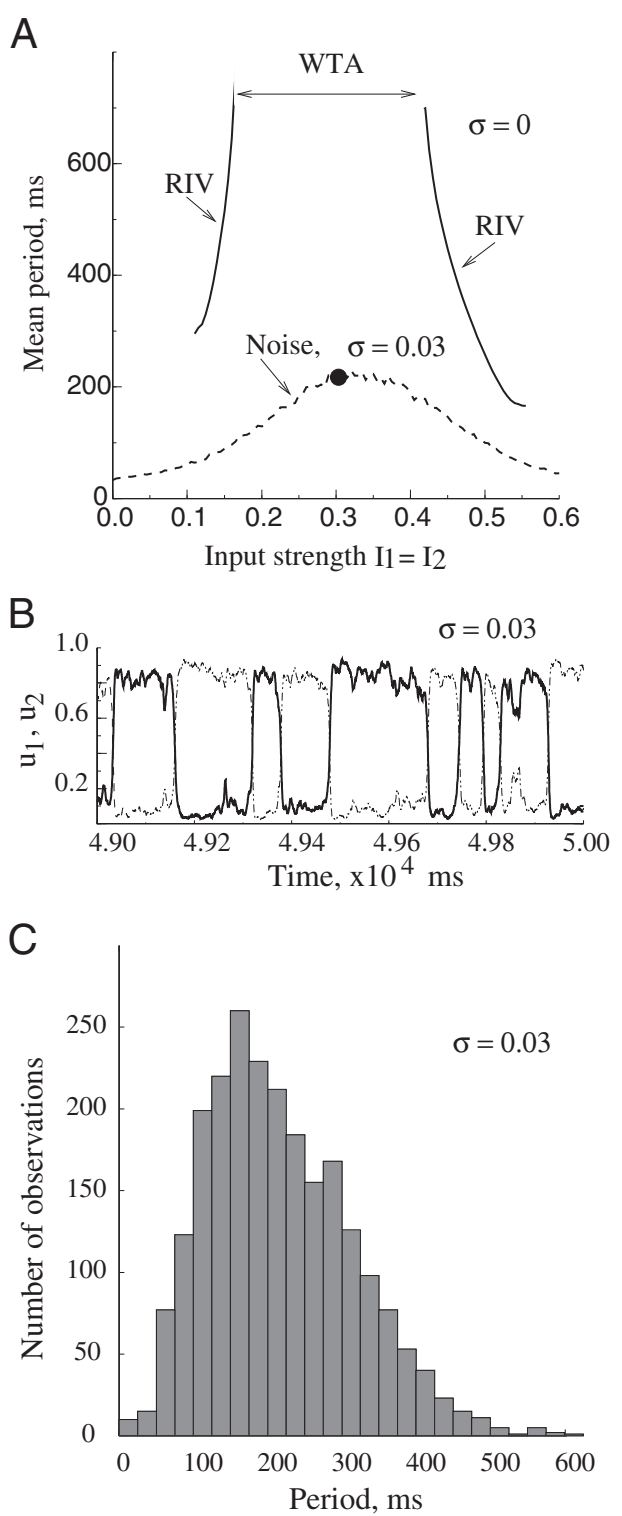

FIG. 6. A: period $T$ of network oscillation vs. input strength for the depression-LC model in the absence (solid line, identical to the one in Fig. 4A) and in the presence (dashed lines) of noise with $\sigma=0.03$. Inhibition strength parameter is $\beta=0.6$. For values of the other parameters, see METHODS. $B$ : typical time course of the network activity, calculated for the input strength value $I_{1}=I_{2}=0.3$ (marked by a filled circle in $A$ ) and noise strength $\sigma=0.03$. $C$ : distribution of the periods durations for this model, calculated for the input strength value $I_{1}=I_{2}=0.3$ and noise strength $\sigma=0.03$. Standard deviation over mean ratio is 0.45 .

simultaneously active at a low level. Thus in general, period depends nonmonotonically on stimulus strength with a winnertake-all behavior that separates the two oscillatory regimes that we have called increasing duration (ID) and decreasing duration (DD).We emphasize that the presence of the winner-takeall and ID regimes is common to all mutual-inhibition models that we considered.

We find that the existence of the winner-take-all and ID regimes is controlled by the strength of inhibition between two neuronal populations. The strength of inhibition is controlled by $\beta$ and thus it is this parameter that directly affects whether there will be an increasing duration regime (as well as a nonoscillatory regime). Our results indicate that there is a 
rather narrow range of $\beta$ values where models show a monotonic behavior, that is, a decreasing period of oscillations as stimulus strength is increased from zero to large values. For example, for the depression-LC, $\beta$ has to be $<0.47$ to obtain such monotonic behavior. Increasing $\beta$ by only $25 \%$, to a value of 0.6 , gives rise to the emergence of an increasing duration regime for stimulus strengths between 0.11 and 0.16 and a nonoscillatory regime for stimulus strengths between 0.16 and 0.41 (Fig. 4). It should also be noted that, because the increasing duration behavior emerges for low-intensity stimuli, it is not possible to eliminate nonmonotonic behavior by invoking mechanisms of gain control that keep the stimulus strength within a limited, prespecified range. Such mechanisms are reasonable to hypothesize at the high end of stimulus strength, i.e., as a means to ensure that the model does not receive inputs that are stronger than what might be considered neurobiologically plausible. However, lowering the stimulus strength gradually to zero is a natural process that need not be (and probably cannot be) circumvented by gain control mechanisms and it is in that range that the new, previously unreported behavior is observed for $\beta$ values outside the narrow range we have characterized. In this study we investigated the occurrence of multiple modes of behavior over ranges of stimulus intensity as the strength of inhibition was varied. More generally, we expect that multiple modes would also be found as other system parameters are varied.

\section{Relevance to experimental work on binocular rivalry}

Experimental observations in binocular rivalry phenomena led to the formulation of hypotheses known as Levelt's Propositions (Levelt 1968). They state that during rivalry: (I) "increase of the stimulus strength in one eye will increase the predominance of the stimulus"; (II) "increase of the stimulus strength in one eye will not effect dominance length for the same eye"; (III) "increase of the stimulus strength in one eye will increase the alternation frequency"; and (IV) "increase of the stimulus strengths in both eyes will increase the alternation frequency." The presence of the increasing duration (ID) regime in the models stands in contrast to Levelt's Proposition IV. However, although Levelt's Propositions I through III were tested in several later studies (e.g., Bossink et al. 1993; Leopold and Logothetis 1996; Mueller and Blake 1989), this has not been the case for Proposition IV. Levelt (1968) formulated his Proposition IV based on previous experiments (Alexander 1951; Alexander and Bricker 1952; Breese 1909; Kaplan and Metlay 1964) that were limited compared with what can be done experimentally nowadays in terms of both stimulus generation and analysis. What is needed to test our predictions are data about mean dominance durations as a function of contrast over a wide range. However, the quantity measured in the experiments that Levelt relied on was the number of cycles per unit time, not the average dominance durations. Many of the stimuli produced, in addition to periods of exclusive dominance of one of the eyes, also periods of "patchy" or mixed states, and therefore the number of cycles per unit time do not provide full information about dominance durations (a reanalysis could examine the latter quantity, but the original data are not available). More recently, Hollins (1980) measured dominance durations as a function of contrast for $1^{\circ}$ sinusoidal gratings. However, the data he presents
(Table 1) for two observers are inconclusive, showing different trends for different observers and eyes. O'Shea et al. (1994) present data from two observers where dominance durations decrease (albeit moderately) as the luminance of two rivaling sinusoidal gratings is increased from scotopic to photopic levels, but it is not clear how such changes in luminance are related to the more common way of changing stimulus strength (contrast at photopic levels). In recent years, as interest in binocular rivalry has been growing, there have been many studies with sophisticated manipulations of context, ocular element grouping, eye of origin, adaptation, and more (Bonneh et al. 2001; Kovacs et al. 1996; Silver and Logothetis 2004; Sobel and Blake 2002; Suzuki and Grabowecky 2002). Such manipulations are difficult to relate to simplified models such as those studied here, which use homogeneous neural subpopulations to represent the abstract concepts of "competing percepts." In contrast, there is a dearth of modern studies providing information about how simple parameters such as stimulus contrast affect the basic observables of dominance duration mean and variance. Furthermore, electrophysiological recordings and brain imaging techniques now also provide an opportunity to observe the activity levels of the competing populations. Testing how these new observables are affected by manipulation of stimulus strength can provide further constraints to distinguish between different models (see, e.g., Polonsky et al. 2000). Therefore our theoretical findings call attention to the importance of testing Levelt's Proposition IV, which is more than thirty, by performing further experiments with modern stimulus presentation and measurement techniques over wide ranges of stimulus strength (Brascamp et al. 2006).

\section{System's behavior when the stimulus strength to the two populations is asymmetrical}

We may also consider the behavior of the system when the stimulus to the two populations is asymmetrical; i.e., in the context of binocular rivalry, the input to one eye remains constant, whereas the other one changes (thus working within the conditions of Levelt's Propositions I through III). In the case of the gain function being a Heaviside step function, arguments similar to those presented earlier in section Release and escape as mechanisms for generating oscillations in neuronal competition models allow us to develop an intuition on how the dominance times of the two populations change as the input to only one of them changes.

In the adaptation-LC model with the Heaviside gain function, in the case where release is operative, the dominance time of one population is defined by the amount of time this population spends in the "up" state, which in turn is defined by the strength of the input to this population. We can apply the same reasoning as we did for the common stimulus strength dependency. Increasing the input to one population increases the dominance time of this same population, whereas the dominance time of the other population remains virtually unchanged. This behavior contradicts the conclusions of Levelt's Propositions I through III.

To the contrary, in the escape mechanism, the dominance time of one population is defined by the amount of time the other population spends in the "down" state, which is defined by the strength of the input to that population. Decreasing the 
input to the population increases the dominance time of the other population, whereas the dominance time of the affected population remains roughly the same, in agreement with Levelt's Propositions I through III.

Similar arguments can be made in the case of the depression-LC model with the Heaviside gain function. Escape mechanism of producing oscillations leads to both DD behavior and satisfaction of Levelt's Propositions I through III.

In the case of the gain function taken to be a Heaviside step function, our intuitive predictions are supported by the results of the numerical simulations (not shown here). Whenever the DD behavior of the period versus common stimulus strength dependency is realized, and Levelt's Proposition IV is satisfied, Levelt's Propositions I through III are satisfied as well. In contrast, if Levelt's proposition IV is not satisfied (ID branch of the period vs. common stimulus strength dependency), we find that Levelt's Propositions I through III are also not satisfied. In the case of the smooth sigmoid gain function, more detailed mathematical analysis is necessary to test the generality of these observations.

\section{Oscillations do not require recurrent excitation}

In the classical firing rate models that are used to describe network oscillations (e.g., Wilson and Cowan 1972), rhythmogenesis is realized by recurrent excitation and slow inhibitory feedback. In three of the models that we studied (W, adaptation-LC, and depression-LC), it is the cross-inhibition that provides competition between two populations; no recurrent excitation is required for the system to oscillate. Oscillations are realized, instead, by the inclusion of a slow negative feedback.

Note, nevertheless, that one of the models we studied does include recurrent excitation-the LC model. As seen from Fig. $5 A$, this model demonstrates all the variety of behaviors observed in the models without the recurrent excitation. We studied the effect of adding recurrent excitation to the $\mathrm{W}$ model as well and, again, we were able to find (not shown) a range of cross-inhibition strength parameter where winner-take-all and increasing duration oscillatory regimes were present. We conclude that the inclusion of the recurrent excitation does not prevent the model from exhibiting multiple modes of behavior that are not observed experimentally.

Inclusion of recurrent excitation may, in fact, have negative consequences for models describing binocular rivalry. The positive feedback from recurrent excitation can lead to rhythmogenesis within an isolated population, that is, the activity of a neuronal unit that is not connected to other units can oscillate when presented with a stationary stimulus. This behavior, to the best of our knowledge, has not been reported experimentally. In addition, as noted by Wilson (2003), in the original W model increase in the strength of the recurrent excitation leads to the disappearance of rivalry.

\section{Competition models in relation to central pattern generator (CPG) models}

Reciprocal inhibition architecture, found in models of binocular rivalry, is also used in the modeling of CPGs (Calabrese 1995; Getting 1988; Skinner et al. 1994). Models from these two different contexts have major components that share qual- itative features. These components may include an autocatalytic process, either synaptic by recurrent excitation or intrinsic by voltage-gated persistent inward currents, and slow negative feedback processes, such as synaptic depression and/or intrinsic adaptation. We noted in the preceding paragraph that recurrent excitation is not essential for rivaling oscillations. Similarly, CPG rhythmicity is possible without such autocatalysis. A recent firing rate CPG model by Taylor et al. (2002) has synaptic depression as a slow negative feedback process and does not include recurrent excitation. Although our focus is binocular rivalry, we conducted some exploratory simulations of this model and found that it exhibits four of the five regimes of behavior as in models of binocular rivalry. We used the parameters as given by Taylor et al. (2002) (with $\mathrm{W}$ and $\tau$ in the range for their Fig. 5) and varied the stimulus intensity $b$, current injected into a cell, over an extended range. As $b$ was decreased from a starting high value we found fusion at high activity levels, then a regime of oscillations with the period increasing as $b$ decreases, then winner-take-all dynamics, and finally fusion at low activity levels. Curiously, the nonoscillating states (not shown in Fig. 5 of Taylor et al. 2002) may, in some cases, coexist with the "rivaling" oscillations. The possibility of such overlapping of region II with I or III could be a valuable or problematic feature. Moreover, with modest variation of parameters this model develops an increasing duration regime, with or without the decreasing duration regime; e.g., by shifting the sigmoid function $\sigma(u)$ rightward along the $u$-axis, no more than one unit, in the $u_{i}$ but not $d_{i}$ dynamics (also, $w=5, \tau=16$ ). We conjecture that our characterization of the multiple behavioral regimes (up to four and five) as stimulus intensity is varied also applies to some other CPG models, and we wonder how this relates to experimental observations in such systems.

\section{Two types of slow negative feedback: subtractive and divisive}

We considered several realizations of the slow processes in our models. One is subtractive adaptation, used in the LC and adaptation-LC models; the second is divisive adaptation in the W models; and finally synaptic depression in the LC and depression-LC models. As mentioned in METHODS, even though divisive adaptation and synaptic depression are implemented mathematically very differently, they both act to decrease the slope of the gain function for the activity variable, as opposed to the subtractive adaptation, which acts to shift the gain function threshold. A computationally relevant distinction is thus between subtractive and divisive slow processes, the former for subtractive adaptation and the latter for both divisive adaptation and synaptic depression. We notice that in the models with divisive realization of the slow process, the increasing duration behavior is less pronounced. As seen from Figs. 3 and 4, the range of the left (increasing duration) regime of the period versus stimulus strength dependency is less than the range of the right (decreasing duration) regime. In the models with a subtractive slow process, the DD and ID regimes have comparable ranges (Fig. 5), although the range of the DD regime in this case is larger than the range of the DD regime in the divisive slow process models (about 0.4 in the LC and adaptation-LC model vs. 0.15 in the depression-LC model; see Figs. 4 and 5). Similar results were obtained by Tabak et al. 
(2005) in studying rhythmogenic behavior in excitatory networks. In their models, the dynamic range of oscillations in the systems with a subtractive mechanism of slow process is larger than in the systems with divisive mechanism. At the same time, we find that a relatively small change in the cross-inhibition strength parameter $\beta$ in the divisive slow process models will produce a transition from monotonic to nonmonotonic behavior; for subtractive slow process models, even a large change in $\beta$ does not yield monotonic (DD-only) behavior. Because both Laing and Chow (2002) and Wilson (2003) discuss only a single model (each), no comparison between different implementations of the slow negative feedback processes was made there. In Taylor et al. (2002), effects of synaptic depression versus adaptation are discussed, but not in the context of the oscillation period versus input current dependency.

\section{Effects of noise on firing rate models of rivaling behavior}

For the deterministic models (both the original W and LC models, and adaptation-LC and depression-LC models) the response becomes steady after a transient period after stimulus introduction; the oscillations, once established, are regular. Experimentally, however, the observed behavior is nondeterministic. During rivalry, the dominance times of each percept are randomly distributed (Fox and Herrmann 1967; Logothetis et al. 1996). We simulated such randomness in all models by introducing input noise. Not unexpectedly, the winner-take-all regime is not sustained with stochastic input. Instead, in the range of parameters where the winner-take-all regime existed in the noise-free model, the system with noisy input shows random rivaling alternations. However, the increasing duration dynamics persists and the ranges of the decreasing duration and increasing duration regimes are of the same order. We found that even in some noise-free cases where weakened inhibition had reduced or eliminated ID behavior (e.g., Fig. 4, $C$ and $F$ ) noisy input restored it (not shown). Although we induced randomness by direct introduction of noisy input (as some other studies had done; see, e.g., Freeman 2005; Lehky 1988), in contrast, random behavior emerged in the deterministic spiking neuron model of Laing and Chow (2002) because of finite-size effects: a small number of units or connections (if coupling is sparse). Importantly, even in the presence of noise the alternations in the models that we are studying are driven by a slow process. Although competition models that are noise-driven have been introduced as well (see, e.g., Freeman 2005; Lumer 1998), we do not consider them here, and these studies themselves did not look at the effect of simultaneously varying the strength of both stimuli on the mean durations.

\section{Other architectural issues}

Newly predicted types of behavior that appear in the simplified two-unit $\mathrm{W}$ and LC, adaptation-LC, and depression-LC rate models are also observed in a spatially distributed model for binocular rivalry. This firing rate model (R. Curtu et al., unpublished observations) assumes two homogeneous populations of neurons, excitatory $(E)$ and inhibitory $(I)$, distributed on a one-dimensional ring network. For some parameter range, as the equal strength of stimuli decreases, the patterns in the network change from both populations being highly active, to an alternation in activity between populations with the period decreasing as the stimulus strength increases, to a winner-takeall regime with only one of the two populations is active forever, then oscillations with the period increasing as the stimulus strength increases, followed by the regime when both populations are inactive.

The presence of the separate inhibitory populations makes the $\mathrm{W}$ model architecturally different from the LC and adaptation-LC and depression-LC models. Note, however, that the timescales for the firing rate $\tau$ and for the inhibition $\tau_{I}$ are both much shorter than the timescale of adaptation $\tau_{H}$; in addition, $\tau_{I} \ll \tau$. Consequently, the inhibitory population tracks the excitatory population almost instantaneously. This means $E_{i}(t)=I_{i}(t)$ in the $E q .1$ system, i.e., that mathematically, the (fast) cross-inhibition can be effectively reduced to being direct, in the same way as it is in the LC, adaptation-LC, and depression-LC models. We observe the same qualitative behavior in a modified version of the $\mathrm{W}$ model with direct inhibition as we did in the original $\mathrm{W}$ model. We conclude that although having distinct excitatory and inhibitory populations in a neuronal competition model is more biologically plausible (in keeping with Dale's principle; Nicoll and Malenka 1998), from a computational perspective the model with indirect inhibition is equivalent to a model with direct inhibition.

\section{Dynamical characteristics common to neuronal computation models}

In conclusion, for all of the competition models that we studied we find additional behaviors that differ qualitatively from those previously reported. Earlier studies described alternations for which the period only decreases as stimulus strength increases. We find the existence of an oscillatory regime in which the period increases as stimulus strength increases, as well as a winner-take-all regime. Although it is easier in some of the models to obtain decreasing dominanceonly dynamics by carefully choosing parameter values (in particular, by reducing the strength of the cross-inhibition), we find common to all the models that we tested very robust nonmonotonic dependency of dominance period on stimulus strength: increasing dominance and decreasing dominance branches, sometimes separated by a winner-take-all regime. Moreover, in the presence of noise, alternations become random, winner-take-all behavior converts to alternation, and the mean dominance period depends continuously and nonmonotonically on stimulus strength, first increasing and then decreasing. This behavior stands in contrast with empirical findings summarized in Proposition IV of Levelt (1968). However, this work is more than thirty years old and surprisingly there are no reports of subsequent studies that duplicate the findings. Our theoretical predictions therefore call for experiments to reexamine with contemporary stimulus presentation and measurement techniques the dependency of the properties of rivalry dynamics on stimulus intensity, in particular to consider a broad range of stimulus strengths. If indeed dominance period is strictly monotonic, decreasing with stimulus strength, then a critical reconsideration of this class of mechanistic models (competitive inhibition with slow negative feedback for alternation) may be in order.

\section{A C K N OW LED G M EN T S}

We thank R. Moreno-Bote and R. O'Shea for helpful discussions. 


\section{G R A N T S}

This work was supported by the National Eye Institute Grants EY07-158-03 to A. Shpiro and EY-14030 to N. Rubin.

\section{REFERENCES}

Alexander LT. The influence of figure-ground relationships in binocular rivalry. J Exp Psych 41: 376-381, 1951.

Alexander LT, Bricker PD. Figure-ground contrast and binocular rivalry. $J$ Exp Psych 44: 452-454, 1952.

Blake R. A neural theory of binocular vision. Psychol Rev 96: 145-167, 1989.

Bonneh Y, Sagi D, Karni A. A transition between eye and object rivalry determined by stimulus coherence. Vision Res 41: 981-989, 2001.

Bossink CJH, Stalmeier PFM, De Weert ChMM. A test of Levelt's second proposition for binocular rivalry. Vision Res 33: 1413-1419, 1993.

Brascamp JW, van Ee R, Noest AJ, Jacobs RHAH, van den Berg AV. The time course of binocular rivalry reveals a fundamental role of noise. $J$ Vis 6 : 1244-1256, 2006.

Breese BB. Binocular rivalry. Psychol Rev 16: 410-415, 1909.

Calabrese RL. Half-center oscillators underlying rhythmic movements. In: The Handbook of Brain Theory and Neural Networks, edited by Arbib MA. Cambridge, MA: MIT Press, 1995, p. 444-447.

Fox R, Herrmann J. Stochastic properties of binocular rivalry alternations. Percep Psychophys 2: 432-436, 1967.

Freeman AW. Multistage model for binocular rivalry. J Neurophysiol 94: 4412-4420, 2005.

Getting PA. Comparative analysis of invertebrate central pattern generators. In: Neural Control of Rhythmic Movements in Vertebrates, edited by Cohen AH, Rossignol S, Grillnet S. New York: Wiley, 1988, p. 101-127.

Grossberg S. Cortical dynamics of three-dimensional form, color, and brightness perception, II: binocular theory. Percep Psychophys 41: 117-158, 1987.

Guckenheimer J, Holmes P. Nonlinear Oscillations, Dynamical Systems, and Bifurcations of Vector Field. New York: Springer-Verlag, 2002.

Hollins M. The effect of contrast on the completeness of binocular rivalry suppression. Percep Psychophys 27: 550-556, 1980.

Kalarickal GJ, Marshall JA. Neural model of temporal and stochastic properties of binocular rivalry. Neurocomputing 32-33: 843-853, 2000.

Kaplan IT, Metlay W. Light intensity and binocular rivalry. J Exp Psych 67: 22-26, 1964.

Kovacs I, Papathomas TV, Yang M, Feher A. When the brain changes its mind: interocular grouping during binocular rivalry. Proc Natl Acad Sci USA 93: 15508-15511, 1996.

Lago-Fernandez LF, Deco G. A model of binocular rivalry based on competition in IT. Neurocomputing 44-46: 503-507, 2002.

Laing CR, Chow CC. A spiking neuron model for binocular rivalry. J Comput Neurosci 12: 39-53, 2002.

Lehky SR. An astable multivibrator model of binocular rivalry. Perception 17: 215-228, 1988.

Leopold DA, Logothetis NK. Activity changes in early visual cortex reflect monkeys' percepts during binocular rivalry. Nature 379: 549-554, 1996.
Levelt WJM. On binocular rivalry. In: Minor Series 2. Psychological Studies. The Hague: Mouton, 1968.

Liu L, Tyler CW, Schor CM. Failure of rivalry at low contrast: evidence of a suprathreshold binocular summation process. Vision Res 32: 1471-1479, 1992.

Logothetis NK, Leopold DA, Sheinberg DL. What is rivaling during binocular rivalry? Nature 380: 621-624, 1996.

Lumer ED. A neural model of binocular integration and rivalry based on the coordination of action-potential timing in primary visual cortex. Cereb Cortex 8: 553-561, 1998.

Matsuoka K. The dynamic model of binocular rivalry. Biol Cybern 49: 201-208, 1984.

Moreno-Bote R, Parga N. Role of synaptic filtering on the firing response of simple model neurons. Phys Rev Lett 92: 0281021-0281024, 2004.

Mueller TJ, Blake R. A fresh look at the temporal dynamics of binocular rivalry. Biol Cybern 61: 223-232, 1989.

Naka KI, Rushton WA. S-potentials from colour units in the retina of fish (Cyprinidae). J Physiol 185: 536-555, 1966.

Nicoll RA, Malenka RC. A tale of two transmitters. Science 281: 360-361, 1998.

O'Shea RP, Blake R. Binocular rivalry and fusion under scotopic luminances. Perception 23: 771-784, 1994.

Polonsky A, Blake R, Braun J, Heeger DJ. Neuronal activity in human primary visual cortex correlates with perception during binocular rivalry. Nat Neurosci 3: 1153-1159, 2000.

Risken H. The Fokker-Planck Equation (2nd ed.). Berlin: Springer-Verlag, 1989.

Silver MA, Logothetis NK. Grouping and segmentation in binocular rivalry. Vision Res 44: 1675-1692, 2004.

Skinner FK, Kopell N, Marder E. Mechanisms for oscillation and frequency control in reciprocally inhibitory model neural networks. J Comp Neurosci 1: 69-87, 1994.

Sobel KV, Blake R. How context influences predominance during binocular rivalry. Perception 31: 813-824, 2002.

Stollenwerk L, Bode M. Lateral neural model of binocular rivalry. Neural Comput 15: 2863-2882, 2003.

Suzuki S, Grabowecky M. Evidence for perceptual "trapping" and adaptation in multistable binocular rivalry. Neuron 36: 143-157, 2002.

Tabak J, O'Donovan MJ, Rinzel J. Differential control of active and silent phases in relaxation models of neuronal rhythms. J Comp Neurosci 21: 307-328, 2006.

Taylor AL, Cottrell GW, Kristan WB Jr. Analysis of oscillations in a reciprocally inhibitory network with synaptic depression. Neural Comput 14: 561-581, 2002.

Wang X-J, Rinzel J. Alternating and synchronous rhythms in reciprocally inhibitory model neurons. Neural Comput 4: 84-97, 1992.

Wilson HR. Computational evidence for a rivalry hierarchy in vision. Proc Natl Acad Sci USA 100: 14499-14503, 2003.

Wilson HR, Cowan JD. Excitatory and inhibitory interactions in localized populations of model neurons. Biophys J 12: 1-24, 1972. 